\title{
Using of a microcapillary refractive X-ray lens for focusing and imaging is
}

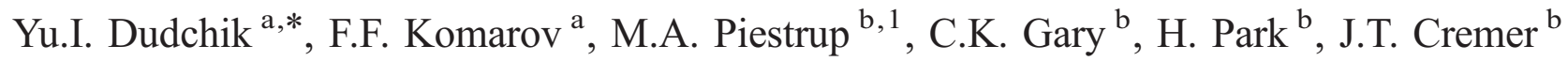 \\ a Institute of Applied Physics Problems, Kurchatova 7, 220064, Minsk, Belarus \\ b Adelphi Technology, 981-B Industrial Rd, San Carlos, 94070, California, United States
}

Received 5 December 2005; accepted 30 May 2007

Available online 7 June 2007

\begin{abstract}
The microcapillary lens, formed by air bubbles in a hollow core glass capillary filled with epoxy, is a novel design of a compound refractive lens for X-rays. The epoxy enclosed between two air bubbles has the form of a biconcave lens and acts as a positive lens for X-rays. Each individual lens is spherical with radius of curvature equal to the inner radius of the capillary. Up to 500 individual biconcave lenses can be formed in a single capillary with diameters from 50 to $500 \mu \mathrm{m}$. Due to the small radius of curvatures that can be achieved, microcapillary lenses typically have shorter focal lengths than those made by compression or injection molding. For example, microcapillary lenses with a focal length about $5 \mathrm{~cm}$ for $8 \mathrm{keV} \mathrm{X}$-rays and 50-micron aperture are readily available.

We have produced a set of lenses in a 200-micron inner-diameter glass capillary with 100-350 individual microlenses and measured their parameters at the Stanford Synchrotron Radiation Laboratory and at the Advanced Photon Source.

Our investigations have also shown that the lenses are suitable for imaging applications with an X-ray tube as a source of X-rays. A simple $\mathrm{X}$-ray microscope is discussed. The microscope consists of a copper anode X-ray tube, X-ray lens and CCD-camera. The object, lens and CCDcamera were placed in-line at distances to satisfy the lens formula. It is shown that the field of view of the microscope is about 1 mm and resolution is equal to $3-5 \mu \mathrm{m}$.
\end{abstract}

(C) 2007 Published by Elsevier B.V.

Keywords: X-ray optics; Compound refractive lenses; X-ray imaging; X-ray microscopy

\section{Introduction}

For hard X-rays the complex refractive index $\mathrm{n}$ for the media is expressed as follows:

$n=1-\delta-i \beta$,

where $1-\delta$ and $\beta$ are real and imaginary parts of refractive index accordingly. Far from edges of absorption the decrement $\delta$ is inversely related to the square of photons energy, and its values lay in a range of $10^{-5}$ to $10^{-7}$. The small value of the

\footnotetext{
This paper was presented at the "18th International Congress on X-ray Optics and Microanalysis" (ICXOM-18) held in Frascati, Rome (Italy), 25-30 September 2005, and is published in the Special Issue of Spectrochimica Acta Part B, dedicated to that conference.

* Corresponding author. Fax: +375 172780417.

E-mail addresses: dudchik@bsu.by (Yu.I. Dudchik), melpie@adelphitech.com (M.A. Piestrup).

URL: http://www.adelphitech.com (M.A. Piestrup).

1 Tel.: +1 650598 9800; fax: +1 6505989400 .
}

decrement leads to the conclusion that it is no any practical interest to focus X-rays by refractive lens [1,2]. The focal length $F$ of such a biconcave spherical lens is determined by the following ratio:

$F=R /(2 \delta)$,

where $R$ is the radius of the lens. The focal length is rather large $(5-10 \mathrm{~m})$ even when the curvature radius of the lens is equal to a few hundred micrometers.

Attempts to reduce focal length of the lens have resulted in creation of a compound refractive lens (CRL) for X-rays with energy $5-30 \mathrm{keV}$, for the first time proposed by Snigirev et al. [3]. The lens consist of a large number (10-300) of biconcave lenses, made of material with a low-atomic weight (beryllium, carbon, polymers, aluminium). The focal length of such a CRL is defined by the following ratio [3]:

$F=R /(2 \delta N)$,

where $N$ is the number of lenses. 


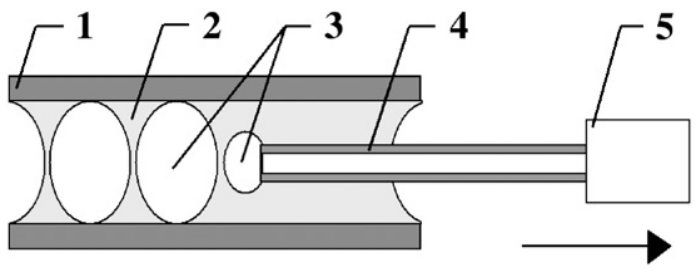

Fig. 1. Schematic view of the setup for lens fabrication. 1 - glass capillary tube; 2 - epoxy; 3 - air bubbles; 4 - injector needle to form air bubbles, 5 cylinder with compressed air.

Eq. (3) shows that the focal length of a compound refractive lens (CRL) is $N$ of times less than the focal length of one lens, and can reach value of $1 \mathrm{~m}$ at $R=0.5 \mathrm{~mm}$. That is quite acceptable for practical applications.

The CRL has been successfully used for focusing synchrotron X-rays [3-8]. The value of the intensity gain in the focal spot depends on source size, and the distance between the lens and the source. A gain of 177 has been achieved [4] for $15 \mathrm{keV}$ $\mathrm{X}$-rays by using a compound refractive lens (CRL), which employs a stack of biconcave parabolic lenses. The CRL designed by Lengeler et al. [4] is also suitable for imaging application yielding submicron resolution.

The idea of compound X-ray lens was advanced by Ref. [9] in which the CRL was fabricated in the form of glass capillary, filled by a large number of epoxy drops. This "microcapillary lens" was used at the synchrotron SPring- 8 for focusing of $18 \mathrm{keV} \mathrm{X}$-rays, and as an objective for an X-ray microscope [10]. The lens consists of a glass microcapillary, filled by $N$ biconcave microlenses. The concave microlenses inside the capillary were formed by putting air bubbles into epoxy. It was shown [11] that the microlenses inside the capillary were spherical, and the bubble radius of curvature was equal to the capillary radius. This dependence of the lens curvature radius on the capillary radius allowed one to decrease the lens' focal length. For example, a CRL comprised of a $200 \mu \mathrm{m}$ diameter capillary filled with 100 air bubbles, had a $13-\mathrm{cm}$ focal length for $8 \mathrm{keV} \mathrm{X}$-rays, as was experimentally demonstrated by Adelphi Technology, Inc. [12] at beamline 2-3 at the Stanford Synchrotron Radiation Laboratory (SSRL). Such short-focallength lenses with $10-\mathrm{cm}$ focal lengths are suitable for imaging not only with synchrotron X-rays, but also with X-rays from laboratory sources of radiation. This was demonstrated in Ref. [13] for $5.4 \mathrm{keV}$ chromium $\mathrm{K} \alpha \mathrm{X}$-rays and in Ref. [14] for Xray from copper anode $\mathrm{X}$-rays tube.

Here we present results of experiments on using a shortfocal-length microcapillary $\mathrm{X}$-ray lens for focusing synchrotron $\mathrm{X}$-rays, and for imaging with 8-keV $\mathrm{Cu} \mathrm{K} \alpha \mathrm{X}$-rays.

\section{Method for the lens fabrication}

The idea of the microcapillary lens is based on a well-known fact, that a drop of a liquid, placed in a glass capillary, takes the form of biconcave lens under action of surface tension forces. As for a lens material, various liquids or polymers can be used. The polymers, as a rule, consist of carbon, hydrogen and

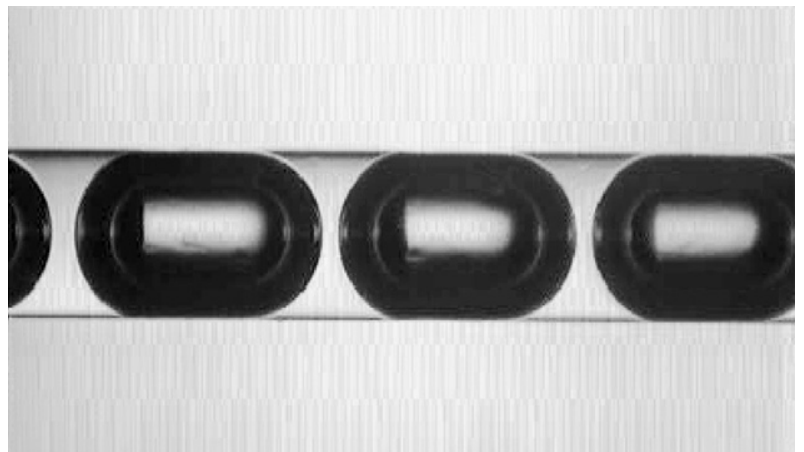

Fig. 2. Photo of the capillary filled by epoxy concave microlenses separated by air bubbles. Capillary diameter is equal to $200 \mu \mathrm{m}$.

nitrogen, each of them is characterized by a low absorption coefficient for 5-30 keV X-rays.

The method of the lens preparation consists $[9,11]$ of the consecutive production of air bubbles inside of capillary 1 , filled by epoxy 2 by use of capillary 4 , which is connected with a cylinder of compressed air 5, as is shown in Fig. 1. The growth of the bubble inside capillary 1 is viewed by a light microscope. When a bubble radius becomes equal to the capillary 1 radius, then capillary 4 moves to few microns distance from the bubble and the process is repeated. The liquid between two bubbles has a form of biconcave lens. The photo of the epoxy lens, made by this method, is shown in Fig. 2. The diameter of the capillary channel is $0.2 \mathrm{~mm}$. It is important that the radius of individual lens is equal to the capillary one, as was shown in Ref. [11]. The epoxy consists of carbon, oxygen, hydrogen and nitrogen which are chemically bonded in proportion $\mathrm{C}_{200} \mathrm{H}_{100} \mathrm{O}_{20} \mathrm{~N}$, and the epoxy density is $1.08 \mathrm{~g} / \mathrm{cm}^{3}$.

An important parameter of the lens is thickness $d$, which depends on the given lens material, and on the diameter of the

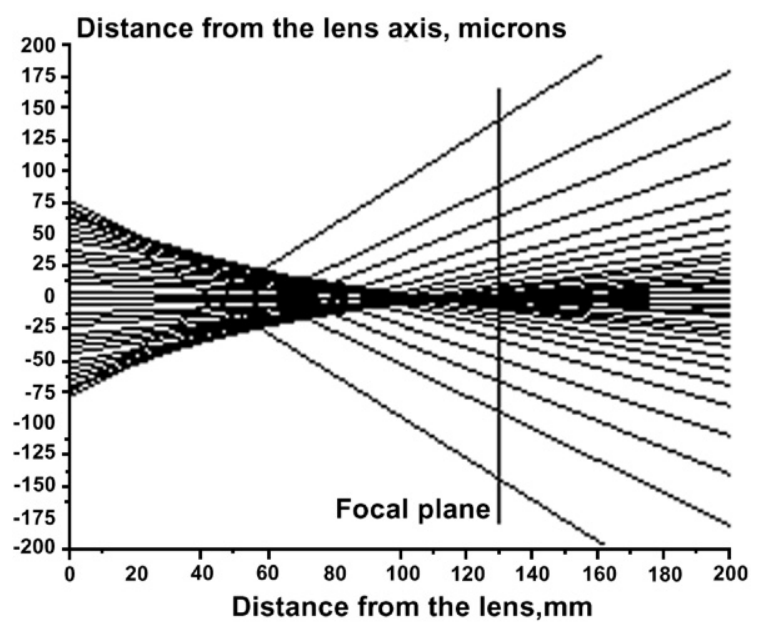

Fig. 3. Paths of X-rays form a focal spot produced by the X-ray lens. The photon energy is $8 \mathrm{keV}$; the radius of the lens is $100 \mu \mathrm{m}$; the number of microlenses lens is equal to 103 . 
Table 1

Measured and calculated parameters of bubble CRLs for SSRL BL 2-3 source (400 mm vertical FWHM $\times 1700 \mathrm{~mm}$ horizontal FWHM) at $16.8 \mathrm{~m}$ distance from CRL

\begin{tabular}{|c|c|c|c|c|c|c|}
\hline Bubble lens designation & $1-1$ & $3-1$ & $3-4$ & $3-3$ & $4-1$ & $4-1$ \\
\hline Photon energy, keV & 12 & 12 & 9 & 8 & 8 & 7 \\
\hline Number of lenses & 90 & 196 & 103 & 93 & 102 & 102 \\
\hline Lens curvature radius, $\mu \mathrm{m}$ & 165 & 250 & 100 & 100 & 100 & 100 \\
\hline Calculated focal length, $\mathrm{cm}$ & 52.8 & 37 & 15.7 & 13.8 & 12.6 & 9.6 \\
\hline Calculated image distance, $\mathrm{cm}$ & 54.5 & 37.8 & 15.8 & 13.9 & 12.6 & 9.7 \\
\hline Measured image distance, $\mathrm{cm}$ & 32 & 36 & 17.5 & 13 & 14 & 10 \\
\hline $\begin{array}{l}\text { Calculated vertical minimum } \\
\text { waist diameter, } \mu \mathrm{m}\end{array}$ & 15.1 & 10.4 & 4.4 & 3.9 & 3.2 & 2.7 \\
\hline $\begin{array}{l}\text { Measured vertical minimum } \\
\text { waist diameter, } \mu \mathrm{m}\end{array}$ & 12.8 & 12 & 3.9 & 4.8 & 2.7 & 4 \\
\hline $\begin{array}{l}\text { Calculated horizontal minimum } \\
\text { waist diameter, } \mu \mathrm{m}\end{array}$ & 64.1 & 44.0 & 18.8 & 16.6 & 13.5 & 11.5 \\
\hline Measured peak transmission, $\%$ & 36 & 30 & 24 & 16 & 27 & 5 \\
\hline $\begin{array}{l}\text { Calculated attenuation aperture } \\
\text { diameter, } \mu \mathrm{m}\end{array}$ & 314 & 262 & 143 & 125 & 119 & 96.7 \\
\hline $\begin{array}{l}\text { Measured attenuation aperture } \\
\text { diameter, } \mu \mathrm{m}\end{array}$ & 321 & 245 & 147 & 150 & 149 & 149 \\
\hline Calculated 2D-gain & 16.6 & 20.0 & 25.6 & 16.9 & 28.9 & 6.0 \\
\hline Measured 2D-gain & 8.9 & 3.5 & 13.4 & - & 25.5 & - \\
\hline
\end{tabular}

capillary channel. We found that for the lens made from epoxy, the lens thickness $d$ might be decreased by $5-10 \mu \mathrm{m}$.

\section{X-ray lens parameters}

The individual epoxy lenses, formed inside of glass capillary, are spherical with the curvature radius equal to the capillary radius. Spherical lenses may be characterized by the following set of parameters: lens focal length $F$, absorption aperture radius $R_{\mathrm{a}}=(2 R / \mu N)^{1 / 2}$, parabolic aperture radius $R_{\mathrm{p}}=\left(2 R^{3} \lambda / \delta N\right)^{1 / 4}$, and the diffraction radius $R_{\text {diff. }}=0.61 \lambda f / R_{\mathrm{a}}$, that characterizes diffraction blurring of the focused beam. The parameters in these equations are the capillary radius $R$, number of lenses $N$, the real part of refractive index $(1-\delta)$, the linear absorption coefficient $\mu$ for the lens material, and the wavelength $\lambda$. The parabolic aperture radius $R_{\mathrm{p}}$ is the central portion of the spherical lens that focuses X-rays to the same point.

Fig. 3 shows trajectories of 8-keV X-ray photons around lens focal spot. The lens has the following parameters: $R=100 \mu \mathrm{m}$, $N=103, d=5 \mu \mathrm{m}$, the microlenses are made from epoxy, where $R, N$ and $d$ are the lens radius, the number of individual lenses, and the minimum (center) lens thickness respectively. The $\mathrm{X}$-ray source is located at a distance $16.81 \mathrm{~m}$ from the lens, which corresponds to the experimental conditions described in paper [12].

As seen from Fig. 3, some rays cross an optical axis before the focal plane due to lens spherical shape. This phenomenon is described mathematically, and the parabolic aperture radius $R_{\mathrm{p}}$ is a good approximation to account for X-rays that are focused to the same point. Calculations show that parabolic aperture radius is $R_{\mathrm{p}}=30 \mu \mathrm{m}$ for the case of the discussed lens $(R=100 \mu \mathrm{m}, N=103)$. The absorption lens aperture radius $R_{\mathrm{a}}$ for the lens is equal to $58 \mu \mathrm{m}$, and theoretically the lens is suitable for focusing $8 \mathrm{keV} \mathrm{X-rays} \mathrm{to} \mathrm{a} \mathrm{diffraction-limited} \mathrm{0.4-}$ micron spot diameter $\left(2 R_{\text {diff }}\right)$.

\section{Measurements}

We fabricated and tested some bubble CRLs arbitrarily designated as $1-1,3-1,3-4,3-3$, and $4-1$. The calculated and measured characteristics of these CRLs are given in Table 1 below. Minimum lens thickness ( $d$, the minimum distance between spheres) was determined by microscopy.

We used beamline 2-3 on the Stanford Synchrotron Radiation Laboratory's (SSRL's) synchrotron. This beamline possesses a double-crystal monochromator that was capable of giving X-rays from 2400 to $30,000 \mathrm{eV}$ with a $5 \times 10^{-4}$ resolution. The approximate source size (full width half maximum, FWHM) was $0.44 \times 1.7 \mathrm{~mm}^{2}$, as specified by SSRL. The experimental apparatus is shown in Fig. 4. The distance from the source to lens was $16.81 \mathrm{~m}$. The X-ray beam size from this source was approximately $2 \times 20 \mathrm{~mm}^{2}$ at the entrance to the experimental station; however, this size was reduced to approximately $0.4 \times 0.4 \mathrm{~mm}^{2}$ by $\mathrm{Ta}$ slits upstream of the monochromator. The CRL was placed in a goniometer head that could be manually tilted in three axes. The lens could also be remotely translated orthogonally ( $x$ and $y$ ) to the direction of the $\mathrm{X}$-ray beam to maximize the X-ray transmission through the lens. An X-ray gas ionization detector was placed after a translatable slit for measuring the X-ray beam profile. This Ta slit was adjusted to below $25 \mu \mathrm{m}$ by using a thin stainless steel shim. It is likely that, as good as the Ta slit was, the jaws are not ideally parallel at these small dimensions, and the slit width was minimally $>3 \mu \mathrm{m}$ when jaws appeared to be entirely closed.

After the slit width was adjusted, the Ta slit was then translated in the $x$ direction across the focused X-ray beam, and its profile obtained. We then manually moved the slits along the $z$-axis of the $\mathrm{X}$-ray beam, measuring its vertical widths by scanning the slits in the $x$ direction across the beam at each location.

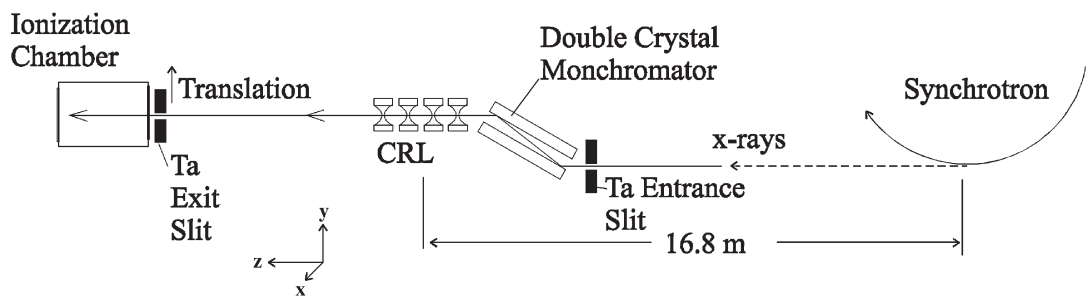

Fig. 4. Experimental apparatus for measuring the profile of a focused X-ray beam and establish the image distance. 
Using these measured widths, we profiled the beam waist as a function of distance from the lens. The FWHM in the vertical direction was plotted as a function of distance from the lens 4-1 for $8 \mathrm{keV}$ photons. The $\mathrm{X}$-ray beam waist converged to minimum size at an approximate distance of $13 \mathrm{~cm}$ from the lens.

The source of diameter $0.44 \mathrm{~mm}$ was focused to a minimum spot FWHM of $5 \mu \mathrm{m}$, at a distance of $13 \mathrm{~cm}$ from the CRL. Thus, the demagnification was $M=.0114$. The spot size in the horizontal direction was measured to be $19 \mu \mathrm{m}$, which is larger, because the imaged source diameter was larger $(1.7 \mathrm{~mm}$, FWHM) in that dimension.

The CRL has an aperture with a Gaussian absorption profile, which causes stronger absorption of the extreme rays passing through the CRL outer radial regions, as compared to rays that pass through the less absorptive central region. The CRL aperture is much smaller than the source size, especially in the horizontal dimension. We obtained the transmission through the CRLs given in Table 1 by narrowing the X-ray beam to $50 \times 50 \mu \mathrm{m}^{2}$ and translating each CRL through the beam, thereby producing transmission profiles of the lenses. The absorption apertures $\left(\mathrm{e}^{-2}\right.$ points, not FWHMs) were obtained from these figures. The calculated and measured peak transmissions (transmission at the lens axis) for the lenses are given in Table 1.

Given the measured transmissions and profiles, we determined the gains of these lenses. Both measured and calculated gains are given in Table 1 for all these lenses. The gain values varied between 3.5 to 25.5 . These gain values are primarily due to the large source size (see Eq. (1)). If the same lens is placed on a beam line using a third generation X-ray source, the gain of the CRL can be substantially larger. These sources can possess spot sizes a factor of 3 smaller (e.g. 0.5 by $0.5 \mathrm{~mm}^{2}$ ). Also, typical distances from insertion devices to end stations can be approximately $51 \mathrm{~m}$ (as was used in Ref. [4]), as compared to $16.8 \mathrm{~m}$ in our experiment, and consequently the increase in the gain is $(51 / 16.8)^{2}$. For these source parameters, the gain at $11 \mathrm{keV}$ from the same lens is 138 , a sizable increase of the intensity over that of the case without a CRL. Although the gains of these CRLs were small, larger gains can be achieved using smaller source sizes, larger CRL apertures, and longer object distances.

\section{X-ray projection imaging}

In-line X-ray projection imaging is based on the using of microspot X-ray tube as a source of radiation. This well-known method of microscopy and microtomography is also suitable for weakly absorbing samples [15]. The system for imaging consists of a quasi-point X-ray source and a CCD-camera.

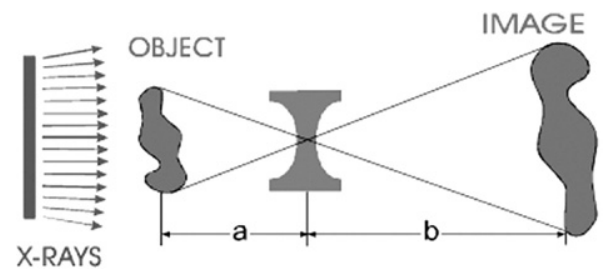

Fig. 5. Schematic of in-line imaging experiment.

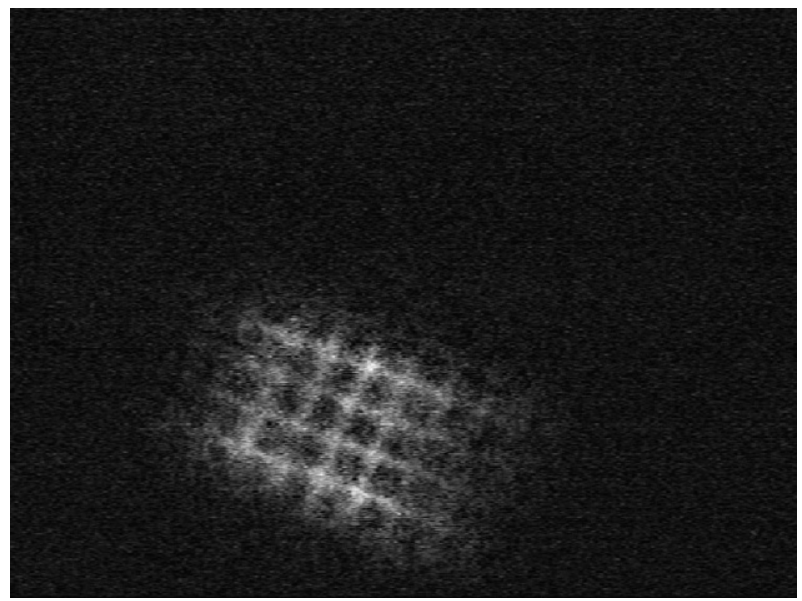

Fig. 6. X-ray image of mesh $\# 400$.

The object for investigation is placed at a distance $R_{1}$ from the source, and the CCD-camera is placed at a distance $R_{2}$ from the sample. The resolution of the method depends on the source size and is about $1 \mu \mathrm{m}$. The magnification is determined as $\left(R_{1}+R_{2}\right) /$ $R_{1}$ and may be 10 or higher. The disadvantage of the method of direct imaging is that the position of the point X-ray source is not stable in time. This disadvantage is remedied by using a refractive lens as an imaging device. In this case there is no limitation to the source size. The scheme of the experimental implementation of this idea is shown in Fig. 5. The sample for investigation was exposed by X-rays produced by a water-cooled copper-anode Xray tube (Russian model \# BCV-17) with tube focal spot of $0.6 \mathrm{~mm} \times 8 \mathrm{~mm}$. The image of the object was recorded by a Marshal B/W CMOS camera with $510 \times 492$ pixels, format $1 / 3$ in., without a glass cover. The object, lens and CCD-camera were placed in-line at distances from one another that satisfied the lens formula (4):

$1 / a+1 / b=1 / f$,

Here, $a$ is the distance from the object to the lens; $b$ is the distance from the lens to CMOS-camera; and $f$ is the lens focal length. The CRL consists of 137 individual spherical, biconcave,

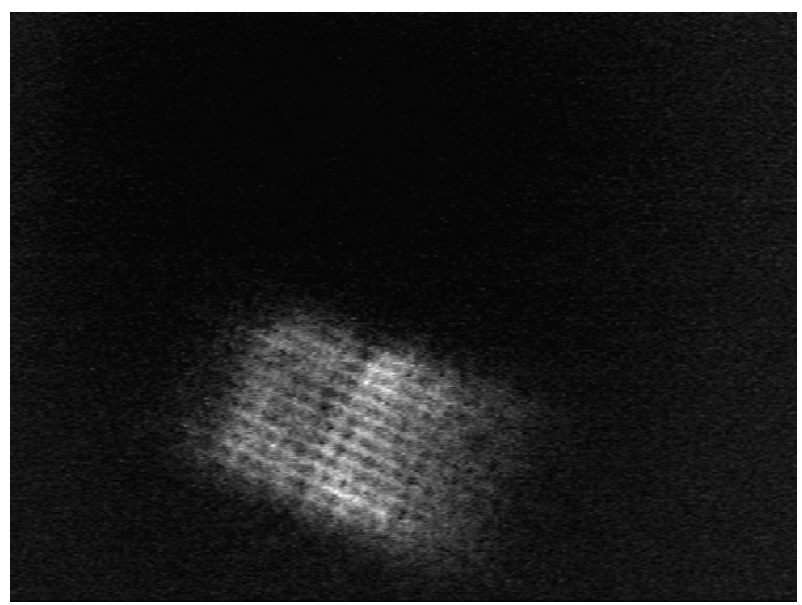

Fig. 7. X-ray image of mesh \# 1000. 
microlenses, each with 100-micron curvature radius $R$. The lens focal length, calculated in accordance to the formula 3 , is $9.6 \mathrm{~cm}$ for $8 \mathrm{keV}$ X-rays. The CRL length is equal to $5.5 \mathrm{~cm}$. Two metal meshes were used as objects for imaging, copper mesh $\# 400$, and gold mesh \#1000. The mesh \#1000 consists of 5-micron thick wires, with a wire center to center separation of $18 \mu \mathrm{m}$. The meshes were placed at distance $a=13 \mathrm{~cm}$ from the lens. The imaging camera were placed at distance $b=51 \mathrm{~cm}$ from the CRL to ensure suitable sharpness of the image. Figs. 6 and 7 are images of metal meshes recorded by the CMOS-camera. The X-ray tube voltage was $32 \mathrm{kV}$ and current $-14 \mathrm{~mA}$. The tube was placed near the object that was imaged.

As it can be seen from Fig. 7 the spatial resolution of the discussed projection X-ray microscope is not more than $5 \mu \mathrm{m}$. Better resolution may be obtained by improving experimental conditions as was shown in the Ref. [14] in which a Photonic Science CCD-camera was used to capture X-ray image, which was imaged by a microcapillary CRL.

\section{Conclusion}

Experiments have shown that a microcapillary refractive $\mathrm{X}$-ray lens is suitable for focusing synchrotron X-rays into micron-sized spots, as well as for imaging applications that use an ordinary X-ray tube as a source of X-rays, and yield at least a 5-micron spatial resolution.

\section{References}

[1] A.G. Michette, Optical Systems for Soft X-rays, Plenum Press, New York, 1986.

[2] S. Suehiro, H. Miyaji, H. Hayashi, Refractive lens for X-ray focus, Nature 352 (1991) 385-386.

[3] A. Snigirev, V. Kohn, I. Snigireva, B. Lengeler, A compound refractive lens for focusing high-energy X-rays, Nature 384 (1996) 49-51.

[4] B. Lengeler, C.G. Schroer, M. Richwin, J. Tummler, M. Drakopoulos, A. Snigirev, I. Snigireva, A microscope for hard X-rays based on parabolic compound refractive lenses, Appl. Phys. Lett. 74 (1999) 3924-3926.

[5] M.A. Piestrup, H.R. Beguiristain, C.K. Gary, J.T. Cremer, R.H. Pantell, R. Tatchyn, Compound refractive lenses for novel X-ray sources, Nucl.
Instrum. Methods Phys. Res., B Beam Interact. Mater. Atoms 173 (2001) $170-177$.

[6] M.A. Piestrup, H.R. Beguiristain, C.K. Gary, J.T. Cremer, R.H. Pantell, Two-dimensional x-ray focusing from compound lenses made of plastic, Rev. Sci. Instrum. 71 (2000) 4375-4379.

[7] H.R. Beguiristain, J.T. Cremer, M.A. Piestrup, C.K. Gary, R.H. Pantell, X-ray focusing using compound lenses made of beryllium, Opt. Lett. 27 (2002) 778-780

[8] J.T. Cremer, M.A. Piestrup, C.K. Gary, R.H. Pantell, Large aperture compound refractive lenses, in: R.O. Tatchyn, Z. Chang, J.-C. Kieffer, J.B. Hastings (Eds.), Fourth-Generation X-ray Sources and Ultrafast X-ray Detectors, Proceedings of SPIE, vol. 5194, SPIE, Bellingham, WA, 2004, p. 56.

[9] Yu.I. Dudchik, N.N. Kolchevsky, A microcapillary lens for X-rays, Nucl. Instrum. Methods Phys. Res., Sect. A, Accel. Spectrom. Detect. Assoc. Equip. 421 (1999) 361-363.

[10] Y. Kohmura, M. Awaji, Y. Suzuki, T. Ishikawa, Yu.I. Dudchik, N.N. Kolchevsky, F.F. Komarov, X-ray focusing test and x-ray imaging test by microcapillary $\mathrm{x}$-ray lens at an undulator beamline, Rev. Sci. Instrum. 70 (1999) 4161-4165.

[11] Yu.I. Dudchik, N.N. Kolchevsky, Fabrication technique and ray tracing calculations for microcapillary X-ray lens, in: A.K. Freund, T. Ishikawa, A.M. Khounsary, D.C. Mancini, A.G. Michette, S. Oestreich (Eds.), Advances in X-Ray Optics, Proceedings of SPIE, vol. 4145, SPIE, Bellingham, WA, 2001, p. 235.

[12] Yu.I. Dudchik, N.N. Kolchevsky, F.F. Komarov, M.A. Piestrup, J.T Cremer, C.K. Gary, R.H. Pantell, Short-focal-length compound refractive lenses for X-rays, in: R.O. Tatchyn, Z. Chang, J.-C. Kieffer, J.B. Hastings (Eds.), Fourth-Generation X-ray Sources and Ultrafast X-ray Detectors, Proceedings of SPIE, vol. 5194, SPIE, Bellingham, WA, 2004, p. 56.

[13] S.A. Pikuz, V.E. Asadchikov, K.M. Chandler, D.A. Hammer, Yu.I Dudchik, N.N. Kolchevsky, F.F. Komarov, M.D. Mitchell, A.V. Popov, T.A. Shelkovenko, R.A. Senin, I.A. Suloev, A.V. Vinogradov, Application of a refractive bubbles-in-capillary X-ray lens to X pinch experiments, Rev. Sci. Instrum. 74 (2003) 2247-2250.

[14] M.A. Piestrup, C.K. Gary, H. Park, J.L. Harris, J.T. Cremer, R.H. Pantell, Yu.I. Dudchik, N.N. Kolchevsky, F.F. Komarov, Microscope using an X-ray tube and a bubble compound refractive lens, Appl. Phys. Lett. 86 (2005) 131104-1-131104-3.

[15] S.C. Mayo, T.J. Davis, T.E. Gureyev, P.R. Miller, D. Paganin, A. Pogany, A.W. Stevenson, S.W. Wilkins, X-ray phase-contrast microscopy and microtomography, Opt. Express 11 (2003) 2289-2302. 